\title{
Columnar Specificity of Microvascular Oxygenation and Volume Responses: Implications for Functional Brain Mapping
}

\author{
Sameer A. Sheth, Masahito Nemoto, Michael Guiou, Melissa Walker, Nader Pouratian, Nathan Hageman, and \\ Arthur W. Toga \\ Laboratory of Neuro Imaging, Department of Neurology, David Geffen School of Medicine at University of California at Los Angeles, Los Angeles, California \\ 90024
}

Cortical neurons with similar properties are grouped in columnar structures and supplied by matching vascular networks. The hemodynamic response to neuronal activation, however, is not well described on a fine spatial scale. We investigated the spatiotemporal characteristics of microvascular responses to neuronal activation in rat barrel cortex using optical intrinsic signal imaging and spectroscopy. Imaging was performed at $570 \mathrm{~nm}$ to provide functional maps of cerebral blood volume (CBV) changes and at $610 \mathrm{~nm}$ to estimate oxygenation changes. To emphasize parenchymal rather than large vessel contributions to the functional hemodynamic responses, we developed an ANOVA-based statistical analysis technique. Perfusion-based maps were compared with underlying neuroanatomy with cytochrome oxidase staining. Statistically determined CBV responses localized accurately to individually stimulated barrel columns and could resolve neighboring columns with a resolution better than $400 \mu \mathrm{m}$. Both CBV and early oxygenation responses extended beyond anatomical boundaries of single columns, but this vascular point spread did not preclude spatial specificity. These results indicate that microvascular flow control structures providing targeted flow increases to metabolically active neuronal columns also produce finely localized changes in CBV. This spatial specificity, along with the high contrast/noise ratio, makes the CBV response an attractive mapping signal. We also found that functional oxygenation changes can achieve submillimeter specificity not only during the transient deoxygenation ("initial dip") but also during the early part of the hyperoxygenation. We, therefore, suggest that to optimize hemodynamic spatial specificity, appropriate response timing (using $\leq 2-3 \mathrm{sec}$ changes) is more important than etiology (oxygenation or volume).

Key words: neurovascular coupling; cerebral blood flow; cerebral blood volume; BOLD fMRI; optical imaging; initial dip; high resolution

\section{Introduction}

The pioneering studies by Mountcastle (1957) and Hubel and Wiesel (1959) demonstrated neurons in primary sensory cortices with similar properties are grouped together in columns. Columnar organization has also been described in higher association areas (Britten, 1998) and may be a general organizational principle of cerebral cortex (Rakic, 2002). Although functional architecture was originally studied with electrophysiological recordings, the role of imaging techniques such as positron emission tomography and functional magnetic resonance imaging ( $\mathrm{fMRI}$ ) is rapidly increasing. Spatial resolution of the latter has increased enough to allow columnar mapping in certain situations (Ugurbil et al., 2003). The ability to resolve individual functional units is essential for developing a deeper understanding of brain organization.

Received 0ct. 5, 2003; revised Nov. 5, 2003; accepted Nov. 8, 2003

This work was supported by grants from the National Institutes of Health (GM08042, MH67432, and MH52083) and the Achievement Rewards for College Scientists Foundation. We thank Dr. Neal Prakash, Allan MacKenzieGraham, and Jacopo Annese for advice on histological procedures.

Correspondence should be addressed to Dr. Arthur W. Toga, Laboratory of Neuro Imaging, David Geffen School of Medicine at University of California at Los Angeles, 710 Westwood Plaza, Suite 4-238, Los Angeles, CA 90024. E-mail: toga@loni.ucla.edu.

DOI:10.1523/JNEUROSCI.4526-03.2004

Copyright $\odot 2004$ Society for Neuroscience $\quad$ 0270-6474/04/240634-08\$15.00/0
Ultimately, the spatial resolution of these imaging techniques is limited by the spatial specificity of the underlying perfusionrelated signals. Activation of a cortical column increases neuronal oxidative metabolism, producing a decrease in tissue oxygenation ("initial dip") spatially restricted to the active column (Malonek et al., 1997; Kim et al., 2000; Thompson et al., 2003). Increased activity generates a vascular response through a variety of putative mechanisms (Villringer and Dirnagl, 1995), leading to an increase in local cerebral blood flow (CBF) proportionately greater than the increase in oxygen metabolism (Fox and Raichle, 1986; Buxton and Frank, 1997). The correspondence between microvascular structures and neural columns (Woolsey et al., 1996) ensures the CBF response is also spatially specific to the activated column (Duong et al., 2001). The influx of oxygenated blood rapidly reverses the transient tissue deoxygenation, leading to a hyperoxygenation that begins in the capillary bed and drains into the venous system. Because the hyperoxygenation is most prominent in medium to large veins, this response is often considered spatially unspecific.

Increased flow also leads to increased cerebral blood volume (CBV), but their dynamic spatiotemporal relationship is not well known. We sought to determine whether the focal CBF increase also leads to a spatially specific CBV increase. Because previous 
studies suggested the volume response is highly vascular and "overspills" active cortex (Frostig et al., 1990; Malonek and Grinvald, 1996), we reasoned reduction of CBV signals from medium to large vessels might improve spatial specificity. We measured CBV changes using optical intrinsic signal (OIS) imaging in the rat barrel cortex system during stimulation of individual neighboring whiskers. To reduce the contribution of large vascular signals, we developed statistical analysis techniques that emphasize parenchymal responses.

Although many studies have found the delayed hyperoxygenation signal is too vascular to resolve submillimeter structures (Malonek and Grinvald, 1996; Kim et al., 2000), it may localize well within the first few seconds of stimulation onset (Menon and Goodyear, 1999). Similarly, Duong et al. (2000) reported the initial dip is spatially specific only within the first $2 \mathrm{sec}$. We, therefore, hypothesized that submillimeter specificity is a function of timing (observing early signals) more than etiology (oxygenation or volume). To investigate this concept further, we examined the spatiotemporal characteristics of functional oxygenation and volume changes with simultaneous OIS imaging and spectroscopy.

\section{Materials and Methods}

Animal preparation. Male Sprague Dawley rats $(n=18 ; 300-450 \mathrm{gm})$ were anesthetized with halothane (5\% induction; $1.5 \%$ maintenance) and maintained at $36.5 \pm 0.5^{\circ} \mathrm{C}$ with a heating blanket (Harvard Apparatus, Holliston, MA). We cannulated the tail artery for blood pressure (BP) and gas monitoring, the femoral vein for anesthesia, and the trachea for artificial ventilation. The skull was thinned, and silicone oil was applied to increase bone translucency. Anesthesia was switched to $\alpha$-chloralose (60 mg/kg bolus, $30 \mathrm{mg} / \mathrm{kg} / \mathrm{hr}$ infusion, i.v.) and pancuronium bromide ( $2 \mathrm{mg} / \mathrm{kg}$ bolus, $1.5 \mathrm{mg} / \mathrm{kg} / \mathrm{hr}$ infusion), and inspired gases were adjusted to maintain physiological variables within the normal range: $\mathrm{BP}, 90-110 \mathrm{mmHg} ; \mathrm{p}_{\mathrm{a}} \mathrm{O}_{2}, 121 \pm 7$ mmHg; $\mathrm{p}_{\mathrm{a}} \mathrm{CO}_{2}, 39 \pm 1$ $\mathrm{mmHg}$ (mean $\pm \mathrm{SE}$ ). Halothane was discontinued for at least $1 \mathrm{hr}$ before imaging.

Optical imaging. Animals were imaged under a SMZ1500 microscope (Nikon). Fiber optics directed white light to the preparation, and reflected light was filtered by a filter wheel (Lambda 10-2; Sutter Instruments, Novato, CA) and captured by a 16-bit CCD camera (Princeton Instruments, Trenton, NJ). We used transmission filters centered at 570 and $610 \mathrm{~nm}$ [full width half maximun (FWHM), $10 \mathrm{~nm}$ ]. Imaging at 570 $\mathrm{nm}$ emphasizes CBV changes, and $610 \mathrm{~nm}$ imaging emphasizes oxygenation changes (Sheth et al., 2003). The field of view covered $5.3 \times 7.2 \mathrm{~mm}$ with a $144 \times 192$ pixel array, providing a $37 \mu \mathrm{m}$ resolution. Imaging was performed at $4 \mathrm{~Hz}$ with a $30 \mathrm{sec}$ intertrial interval, and filters were alternated every trial.

Image analysis and histology. Ratio images were generated by subtracting and dividing a prestimulus baseline image from each image in a trial. Response time courses were calculated within a $1.0 \mathrm{~mm}$ diameter region of interest (ROI) in the trial-averaged data. ROI position was determined by an automated program (Matlab; The Mathworks, Natick, MA) that scanned the ROI across the peak $570 \mathrm{~nm}$ image and chose the position resulting in the greatest signal change. This ROI was applied to the averaged data of both wavelengths.

Functional maps were generated in two ways after Gaussian spatial filtering (three-pixel FWHM). The first method used the common approach of applying absolute thresholds to the ratio images (Chen-Bee et al., 2000). The second method used a technique that reduces the influence of large vascular fluctuations and provides statistical significance thresholds. An ANOVA test was performed at every pixel on a $64 \times 3$ matrix: the pixel value in each of 64 trials at three time points $[t=-3.50$ $\sec$ (prestimulus); $t=$ (peak image- 1$) ; t=$ (peak image)]. Functional representations were displayed as the negative log of the resulting $p$ value map. To identify the peak response area, we selected the 100 most significant pixels $\left(\sim 0.14 \mathrm{~mm}^{2}\right.$; approximately half the surface projection of one barrel). After imaging, at least four fiducial lesions (10 sec, $10 \mu \mathrm{A})$ were made.

Animals were perfused with $0.1 \mathrm{~m}$ phosphate buffer and $4 \%$ paraformaldehyde. The right cortex was removed, flattened, cryoprotected, and cut tangentially in $40 \mu \mathrm{m}$ sections. Sections were stained (Wong-Riley and Welt, 1980), photographed, and aligned to raw optical images in Photoshop (Adobe Systems, San Jose, CA).

Optical spectroscopy. We performed optical spectroscopy and imaging concurrently using a fiber bundle mounted on the microscope eyepiece that directed half the reflected light to a spectrophotometer (SpectraPro 300i; $100 \mu \mathrm{m}$ slit; Acton Research, Acton, MA) and 16-bit CCD camera (Spec-10:400BR, Princeton Instruments). Spectra were acquired from 520 to $653 \mathrm{~nm}$ with $0.5 \mathrm{~nm}$ resolution with timing identical to that of image acquisition.

Reflectance spectra were analyzed using a modified form of the BeerLambert law incorporating optical attenuation attributable to absorption and scattering:

$$
\log \frac{I_{\mathrm{o}}^{\lambda}}{I(t)}=\left(\epsilon_{\mathrm{Hbr}}^{\lambda} \times \Delta[\mathrm{Hbr}](t)+\epsilon_{\mathrm{HbO}_{2}}^{\lambda} \times \Delta\left[\mathrm{HbO}_{2}\right](t)\right) \times l^{\lambda}+\Delta S
$$

where $I_{\mathrm{o}}$ is the prestimulus intensity, $I(t)$ is the intensity time course, $\epsilon$ are extinction coefficients, $l$ is the pathlength through the tissue, $S$ is scattering, and $\Delta[H b x](t)$ is the time course of change in absorbers. The high degree of scattering in biological tissue distorts the $\mathrm{Hb}$ attenuation spectra by significantly increasing the optical pathlength in a wavelengthdependent manner. To account for this pathlength wavelength dependency (superscript $\lambda$ ), we used an in vitro phantom model to simulate the absorption and scattering properties of rat cortex (Sato et al., 2002). The phantom contained rat blood as the absorber and Intralipid (Fresenius Kabi, Clayton, NC), as the scatterer. The blood was diluted to $1 \%(\sim 50$ $\mu \mathrm{M}$ ), the approximate mean value in rat brain (Cooper et al., 1998) and within the range assumed by other studies (Jones et al., 2001; Lindauer et al., 2001). A 1\% Intralipid solution provided a reduced scattering coefficient $\left(\mu_{\mathrm{s}}{ }^{\prime}\right)$ of $1 \mathrm{~mm}^{-1}$, similar to that of brain tissue (Nakai et al., 1997). Although the contribution of scattering to optical attenuation is large, changes in scattering during functional activation are small, because the hemodynamic response and associated flow increase are more likely to affect absorption than scattering (Obrig and Villringer, 2003). We, thus, made the common simplifying assumption that scattering and pathlength wavelength dependency remained constant over time.

If we define $\alpha=\epsilon \times l$ and remove time dependency, Equation 1 becomes:

$$
\log \frac{I_{\mathrm{o}}^{\lambda}}{\mathrm{I}^{\lambda}}=\alpha_{\mathrm{Hbr}}^{\lambda} \times[\mathrm{Hbr}]+\alpha_{\mathrm{HbO}_{2}}^{\lambda} \times\left[\mathrm{HbO}_{2}\right]+S
$$

where $I_{\mathrm{o}}$ is the in vitro reference spectrum with $0 \%$ hematocrit, $\alpha_{\mathrm{Hbr}}=$ $\epsilon_{\mathrm{Hbr}} \times l$, and $\alpha_{\mathrm{HbO} 2}=\epsilon_{\mathrm{HbO} 2} \times l$. By holding hematocrit fixed and measuring the reflected intensity, we calculated $\alpha_{\mathrm{Hbr}}$ and $\alpha_{\mathrm{HbO} 2}$, which represent the effective pathlength-adjusted attenuation spectra of $\mathrm{Hbr}$ and $\mathrm{HbO}_{2}$. The phantom solution was fully oxygenated $([\mathrm{Hbr}]=0)$ by bubbling $100 \% \mathrm{O}_{2}$ and deoxygenated $\left(\left[\mathrm{HbO}_{2}\right]=0\right)$ by allowing yeast ( $0.5 \%$ by weight) to consume $\mathrm{O}_{2}$ and adding $30 \mathrm{mg}$ of $\mathrm{Na}_{2} \mathrm{O}_{2} \mathrm{~S}_{4}$. Scattering was assumed to be wavelength independent, because the wavelengthdependent component (i.e., differential pathlength) was incorporated into the effective attenuation spectra. In vitro measurements were made with the same instrument settings and geometry as in vivo measurements. Functional $\mathrm{Hbr}$ and $\mathrm{HbO}_{2}$ time courses were calculated from Equation 1 using a least-squares analysis and the values of $\alpha_{\mathrm{Hbr}}$ and $\alpha_{\mathrm{HbO} 2}$. Because of potential differences in pathlength scale between the in vitro and in vivo conditions, results are reported as relative changes from baseline.

Experimental paradigm. We investigated spatial specificity $(n=10)$ of oxygenation and volume changes in whisker barrel cortex (Woolsey and Van der Loos, 1970). We rotated the stereotactic frame $\sim 12^{\circ}$ around the rostrocaudal axis to orient barrel cortex normal to the optical axis. All whiskers, except $\mathrm{C} 1$ and $\mathrm{C} 2$, were trimmed. Whisker deflection was provided by a motorized stroker, consisting of a vertical rod attached to a 


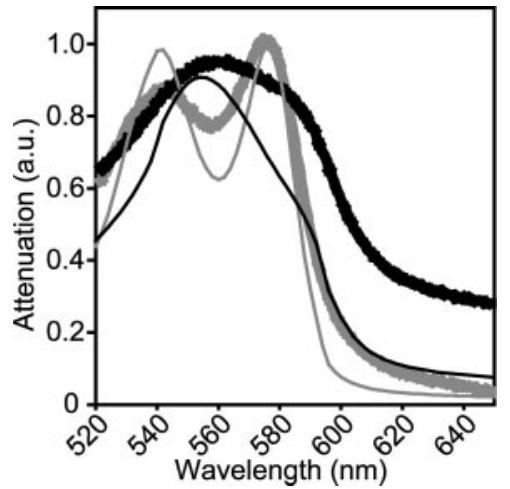

Figure 1. Phantom hemoglobin attenuation spectra. To account for the wavelengthdependent pathlength through highly scattering biological tissue, we measured reflectance spectra from an in vitro phantom simulating the scattering and absorption properties of brain (see Materials and Methods). Phantom attenuation spectra for $\mathrm{HbO}_{2}$ (thick gray line) and $\mathrm{Hbr}$ (thick black line) were distorted relative to textbook extinction coefficients for $\mathrm{HbO}_{2}$ (thin gray line) and $\mathrm{Hbr}$ (thin black line), which do not account for scattering. This difference is attributable to the inclusion of the differential pathlength factor in the highly scattering medium. Phantom and textbook spectra were normalized to facilitate comparison.

motor. Whiskers were deflected $2.8 \mathrm{~mm}$ in $11.5 \mathrm{msec}$ in a rostral-tocaudal direction, measured $\sim 1 \mathrm{~cm}$ from the face. Either $\mathrm{C} 1$ or $\mathrm{C} 2$ was deflected $(2 \mathrm{sec}, 5 \mathrm{~Hz})$ during each trial, for a total of 128 trials (64 per wavelength) for each whisker.

In another eight animals, we studied the effect of response timing and etiology on spatial specificity with concurrent optical imaging and spectroscopy during electrical hindpaw stimulation (Sheth et al., 2003), which allowed us to modulate responses by varying amplitude $(0.4,0.5$, $0.6,0.8,1.0$, and $1.2 \mathrm{~mA}$ at $5 \mathrm{~Hz})$ and frequency $(2,5,10,15$, and $20 \mathrm{~Hz}$ at $0.8 \mathrm{~mA}$ ). Stimulation $(2 \mathrm{sec}$ ) consisted of $1 \mathrm{msec}$ electrical pulses (ISOFlex, Master-8; AMPI, Jerusalem, Israel) to the left hindpaw via two needle electrodes inserted into the plantar surface of the foot and $\sim 10$ $\mathrm{mm}$ away on the medial aspect of the leg. Twelve trials were collected per wavelength and stimulus condition.

\section{Results}

We assessed the spatiotemporal characteristics of functional changes in CBV and oxygenation with high resolution using OIS imaging and spectroscopy. We first confirmed that $570 \mathrm{~nm}$ OIS imaging reflects total hemoglobin ( $\mathrm{Hbt}$ ) and is, therefore, proportional to local changes in CBV. A novel statistical analysis procedure was used to generate functional maps with decreased vascular contribution. These maps demonstrated that volume responses could resolve columnar architecture with submillimeter precision. We provide evidence that optimal hemodynamic spatial specificity is primarily a function of timing rather than response etiology.

\section{In vitro phantom spectra}

The phantom attenuation spectra shown in Figure 1 represent the extinction coefficients of $\mathrm{HbO}_{2}\left(\alpha_{\mathrm{HbO} 2}\right)$ and $\mathrm{Hbr}\left(\alpha_{\mathrm{Hbr}}\right)$ corrected for the wavelength-dependent pathlength. Inclusion of the differential pathlength distorted the phantom spectra relative to textbook absorption coefficients. Textbook spectra isosbestic points are at 522, 549,569, and $586 \mathrm{~nm}$; the $\mathrm{HbO}_{2}$ maxima are at 542 and $577 \mathrm{~nm}$; and the Hbr maximum is at $555 \mathrm{~nm}$. Measured phantom spectra $(0.5 \mathrm{~nm}$ resolution) isosbestic points were at $525,545,570.5$, and $583 \mathrm{~nm}$; the $\mathrm{HbO}_{2}$ maxima were at 545 and $577.5 \mathrm{~nm}$; and the Hbr maximum was at $557.5 \mathrm{~nm}$.

\section{Temporal characteristics}

Whisker stimulation produced monophasic reflectance changes at $570 \mathrm{~nm}$ and biphasic changes at $610 \mathrm{~nm}$ (Fig. 2). The $570 \mathrm{~nm}$

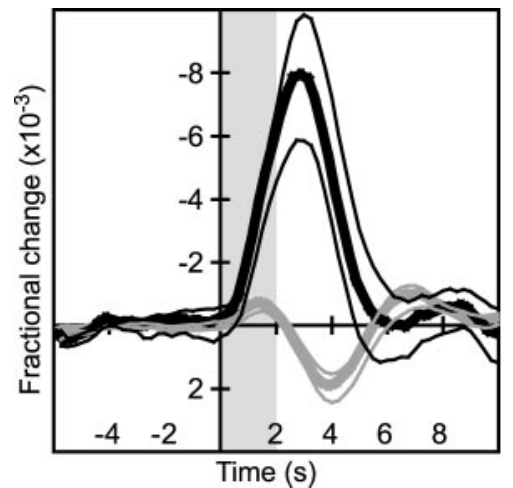

Figure 2. The 570 and $610 \mathrm{~nm}$ response to whisker stimulation. (1 whisker stimulation produced a monophasic reflectance decrease at $570 \mathrm{~nm}$ (thick black line), peaking 2.5-2.75 sec after stimulus onset. The $610 \mathrm{~nm}$ response (thick gray line) was biphasic, with an early negative peak occurring at $1.5 \mathrm{sec}$ and a late positive peak at $4.0 \mathrm{sec}$. Negative changes are plotted upward for convention. Responses to C2 stimulation (data not shown) were similar. The shaded gray region indicates stimulus duration. The average responses are denoted by thick lines, and SEs by thin lines.
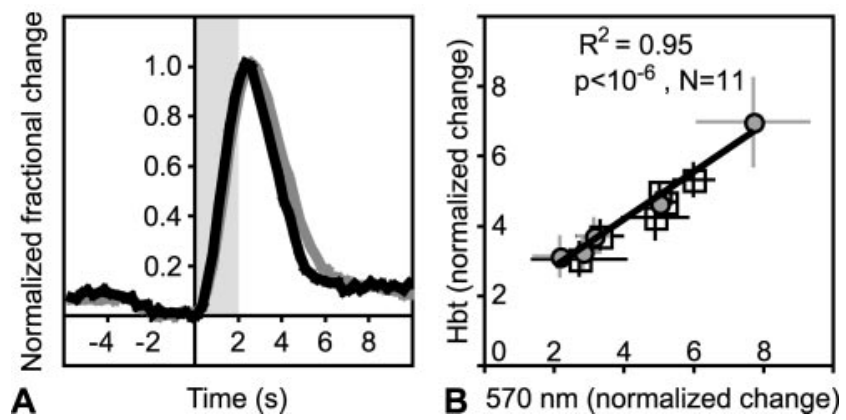

Figure 3. Etiology of $570 \mathrm{~nm}$ OIS responses. A, Normalized time courses of $570 \mathrm{~nm}$ (thick gray line) and $\mathrm{Hbt}$ (thick black line) responses from eight subjects. Both responses have similar latencies, time to peak, and overall profiles. B, Correlation analysis of normalized Hbt versus 570 $\mathrm{nm}$ peak responses to varying stimulation frequency (gray circle) and amplitude (open square) demonstrates strong correspondence between the two responses. Similar temporal profiles and peak magnitude coupling suggest that $570 \mathrm{~nm}$ imaging reflects changes in Hbt. Error bars represent SE.

peak occurred 2.5-2.75 sec after stimulus onset, and the $610 \mathrm{~nm}$ early and late phase peaks occurred at 1.5 and $4.0 \mathrm{sec}$, respectively. Baseline noise (SD of prestimulus time points) was $2.5 \times 10^{-4}$ at $570 \mathrm{~nm}$ and $8.2 \times 10^{-5}$ at $610 \mathrm{~nm}$. The contrast/noise ratio (CNR) (peak magnitude divided by noise) was 30.1 for the 570 $\mathrm{nm}$ response, 7.2 for the $610 \mathrm{~nm}$ early phase, and 22.3 for the 610 $\mathrm{nm}$ late phase. We observed post-response hemodynamic oscillations consistent with other studies (Mayhew et al., 1996; Jones et al., 2001).

Because $569 \mathrm{~nm}$ is an isosbestic point of the oxyhemoglobin $\left(\mathrm{HbO}_{2}\right)$ and deoxyhemoglobin (Hbr) spectra, imaging at $570 \mathrm{~nm}$ is thought to emphasize changes in $\mathrm{Hbt}$, which are proportional to changes in CBV under the assumption of constant hematocrit. The contribution of other wavelengths (attributable to finite width of the bandpass filter) and optical effects (such as scattering) to the imaging signal is a potential confounding factor, however. To estimate its importance, we compared the $570 \mathrm{~nm}$ and Hbt time courses with simultaneous OIS imaging and spectroscopy (Fig. 3). To ensure equal sampling, the imaging response was measured in the same area sampled by the spectroscopic fiber. Both time courses were normalized to their peak value $\left(-2.3 \pm 0.6 \times 10^{-2}\right.$ for $570 \mathrm{~nm}$ and $1.0 \pm 0.1 \times 10^{-2}$ for Hbt; 
mean $\pm \mathrm{SE})$ to facilitate comparison. The $570 \mathrm{~nm}$ response latency (time at which the signal rose $>2 \mathrm{SD}$ above the baseline mean) was $1.0 \mathrm{sec}$ after stimulation onset, and peak was at 2.75 sec. Similarly, Hbt started at $1.25 \mathrm{sec}$ and peaked at $2.75 \mathrm{sec}$. We varied stimulation frequency and amplitude to modulate response size. Figure $3 B$ shows a scatterplot of normalized $570 \mathrm{~nm}$ peak responses versus normalized Hbt peak responses, demonstrating a close correlation $\left(R^{2}=0.95 ; p<10^{-6}\right)$ between the signals. This strong linear relationship, along with similar latencies, time to peak values, and time course profiles, suggests that $570 \mathrm{~nm}$ imaging accurately reflects changes in Hbt. This result is consistent with previous reports that found similarities in spatial characteristics between $570 \mathrm{~nm}$ signals and CBV measured with fluorescent dyes (Frostig et al., 1990). Functional maps (570 nm) are, therefore, taken to represent CBV in this study.

\section{Spatial characteristics}

Figure 4 shows raw cortical images and functional activation maps using both absolute and statistical thresholding techniques for the three responses (monophasic, $570 \mathrm{~nm}$; early and late phases, $610 \mathrm{~nm}$ ) during $\mathrm{C} 1$ and $\mathrm{C} 2$ stimulation in a representative case. The $570 \mathrm{~nm}$ response has not only a larger magnitude than the $610 \mathrm{~nm}$ early phase but also a larger areal extent. Because of this spatial spread, $570 \mathrm{~nm}$ activation maps were thresholded at higher values. This figure highlights the difference between maps generated using absolute reflectance change thresholds and statistically determined thresholds. Absolute reflectance change maps (Fig. 4, left column) tend to accentuate vascular structures. Imaging at $610 \mathrm{~nm}$ emphasizes oxygenation changes, which predominate in draining veins. Venous signals were, therefore, readily apparent in $610 \mathrm{~nm}$ reflectance maps. Similarly, CBVdominant $570 \mathrm{~nm}$ reflectance maps highlight arterioles, in which CBV fractional changes are greatest (Lee et al., 2001). Statistical maps, such as the ANOVA-based maps in this study (Fig. 4, right column), decrease vascular contributions to the functional signal. The magnitude of reflectance changes in vessels can be very large; therefore, vascular signals may linger in reflection change maps even with extensive trial averaging. Because they are not as consistent as parenchymal changes from trial to trial, however, their statistical significance is diminished relative to capillary bed changes. Statistical maps of both 570 and $610 \mathrm{~nm}$ early signals thus emphasize parenchymal changes (Fig. $4 D, F, H, J$ ). Late phase $610 \mathrm{~nm}$ signals are predominantly venous and, therefore, appeared similar using both analyses.

\section{CBV spatial specificity}

To determine the spatial specificity of 570 and $610 \mathrm{~nm}$ early phase responses, we compared statistically defined response areas with $\mathrm{CO}$-stained barrels. Figure 5 shows vertical projections of $\mathrm{C} 1$ and C2 barrels aligned with peak 570 and $610 \mathrm{~nm}$ early phase responses. Peak 570 and $610 \mathrm{~nm}$ early response contours localized well to the barrel projections. The representations of the neighboring columns were distinct using both signals, suggesting that both could accurately resolve submillimeter structures.

To compare 570 and $610 \mathrm{~nm}$ early phase spatial specificity over all 10 subjects, we calculated the center of mass of the two peak response areas. The separation between the responses during $\mathrm{C} 1$ stimulation was $265 \pm 56 \mu \mathrm{m}$ and during $\mathrm{C} 2$ stimulation was $234 \pm 49 \mu \mathrm{m}$, less than the average barrel hollow diameter of 400-500 $\mu \mathrm{m}$ (Welker, 1971). The separation between the representations of $\mathrm{C} 1$ and $\mathrm{C} 2$ was $640 \pm 90 \mu \mathrm{m}$ for the $570 \mathrm{~nm}$ response and $644 \pm 83 \mu \mathrm{m}$ for the $610 \mathrm{~nm}$ early response, also consistent with the interbarrel distance of $\sim 600 \mu \mathrm{m}$.

\section{Vascular point spread}

Hemodynamic response morphology is dictated by vascular architecture (Woolsey et al., 1996; Harrison et al., 2002) and may, therefore, extend beyond neuroanatomical boundaries (Narayan et al., 1995). To investigate the degree of vascular point spread, we compared response magnitudes over the principally stimulated barrel to those over the neighboring barrel (i.e., response to $\mathrm{C} 1$ stimulation over $\mathrm{C} 1$ vs over $\mathrm{C} 2$, and vice versa) in all subjects (Fig. 6 ). Both volume and early oxygenation responses spread beyond the principal barrel but were always significantly smaller in the neighboring barrel. The ratio of principal/neighboring barrel response size was $1.7 \pm 0.3$ for the $610 \mathrm{~nm}$ early phase (C1 and C2). The response ratio for $570 \mathrm{~nm}$ was $1.3 \pm 0.1$ for $\mathrm{C} 1$ and $1.4 \pm 0.2$ for C2. Differences between 610 and $570 \mathrm{~nm}$ response ratios were not significant $(p>0.2)$. Temporal characteristics of responses over the principal and neighboring column were nearly identical within our limits of temporal resolution for all phases of the hemodynamic response.

\section{Spatial specificity and timing}

Spatial specificity of the $610 \mathrm{~nm}$ early negative response has been confirmed with single-unit recordings (Masino et al., 1993; Shoham and Grinvald, 2001), voltage-sensitive dye imaging (Takashima et al., 2001; Slovin et al., 2002), and CO histology (Ts'o et al., 1990; Erinjeri and Woolsey, 2002). Although studies often assume that this response is analogous or even identical to the initial dip, the absorption coefficient of $\mathrm{Hbr}$ is only approximately three times that of $\mathrm{HbO}_{2}$ in the $600 \mathrm{~nm}$ (red light) range. We performed simultaneous optical imaging and spectroscopy to determine the contribution of $\mathrm{Hbr}, \mathrm{HbO}_{2}$, and $\mathrm{Hbt}$ to the $610 \mathrm{~nm}$ signal over time (Fig. 7).

The first $0.75 \mathrm{sec}$ of the $610 \mathrm{~nm}$ response followed Hbr, but whereas Hbr subsequently decreased, the $610 \mathrm{~nm}$ signal continued increasing for another $0.5 \mathrm{sec}$, peaking when $\mathrm{Hbr}$ was well below baseline. The first $0.75 \mathrm{sec}$ of decreased reflectance at 610 $\mathrm{nm}$ thus derived from increased Hbr during the initial dip. The remainder of the response including the peak, however, occurred during the early hyperoxygenation and may be attributable to $\mathrm{Hbt}$ (volume) or $\mathrm{HbO}_{2}$ rising more rapidly than the $\mathrm{Hbr}$ decrease. Across all animals and stimulus presentations (Fig. 7B), the spatially specific $610 \mathrm{~nm}$ early negative phase again incorporated both the initial dip and the early hyperoxygenation. Later hyperoxygenation changes comprised the $610 \mathrm{~nm}$ late phase and were spatially unspecific and vascular.

\section{Discussion}

Our results have two significant implications. First, they suggest the microvascular mechanisms that provide localized CBF changes also lead to localized changes in CBV. This specificity requires reducing the contribution of signals from large vessels, which are usually distant from the site of neuronal activity. Second, we found spatial specificity is predominantly a function of timing, observing early $(<2-3 \mathrm{sec})$ changes, rather than specific response etiology, oxygenation or volume. We discuss these findings and their implications for functional brain mapping.

\section{CBV spatial specificity}

Our results indicate the CBV response is capable of resolving submillimeter functional architecture. CBV spatial specificity is comparable with that of the early oxygenation response, which may be considered a gold standard for high-resolution cortical mapping because of its well-documented specificity. The separa- 

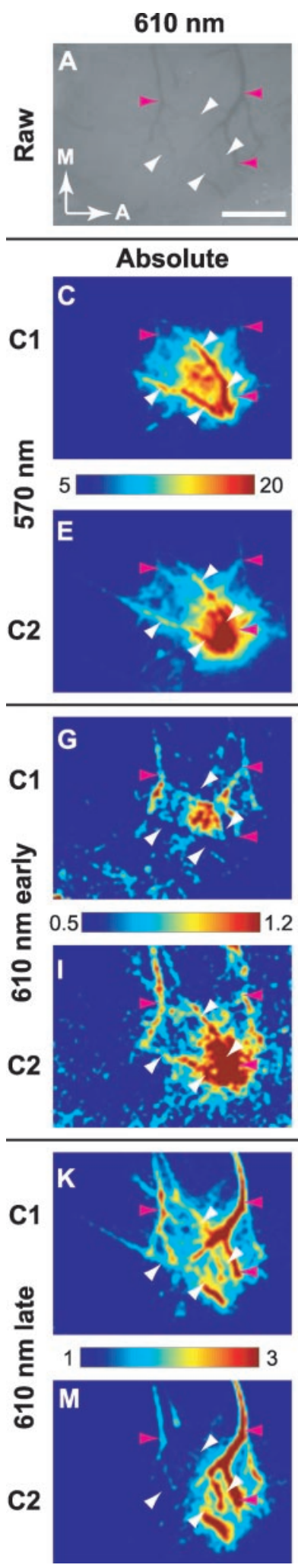

$570 \mathrm{~nm}$

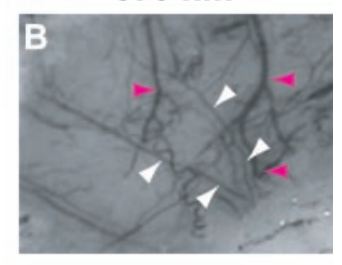

Statistical
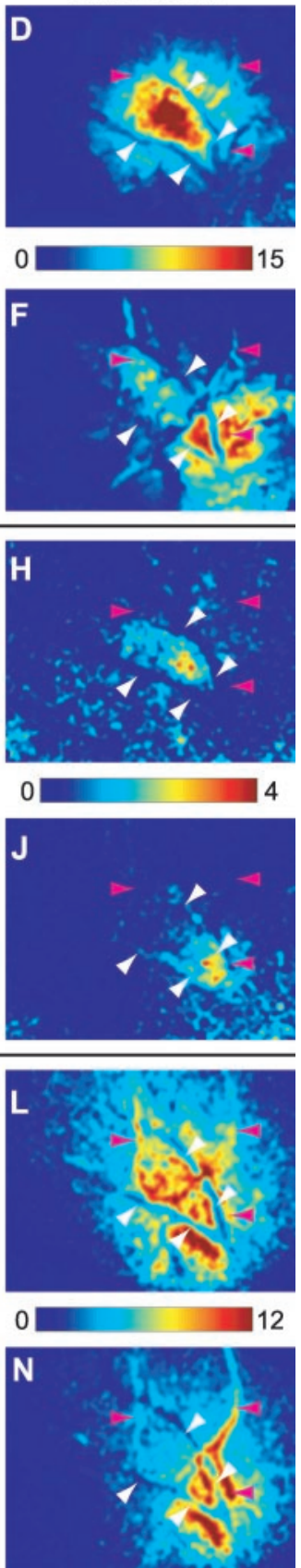

Figure 4. Absolute versus statistical thresholds. The response to C1 and C2 whisker stimulation is shown in a representative subject using absolute (left column) and statistical (right column) thresholds. Raw images of the cortex under $610 \mathrm{~nm}(A)$ and $570 \mathrm{~nm}(B)$ illumination were used to distinguish between surface veins (magenta arrowheads) and arterioles (white arrowheads), because both are equally visible at $570 \mathrm{~nm}$ (showing $\mathrm{Hbt}$ ), but veins are dominant at $610 \mathrm{~nm}$ (emphasizing $\mathrm{Hbr}$ ). $(-F$, The $570 \mathrm{~nm}$ response. $G-J$, The $610 \mathrm{~nm}$ early phase. $K-N$, The $610 \mathrm{~nm}$ late phase. The $570 \mathrm{~nm}$ absolute threshold maps $(C, E)$ highlight activated arterioles showing large reflectance changes. This vascularity is reduced in the statistical map $(D, F)$, which emphasizes changes in the capillary bed. Venous signals evident in absolute threshold

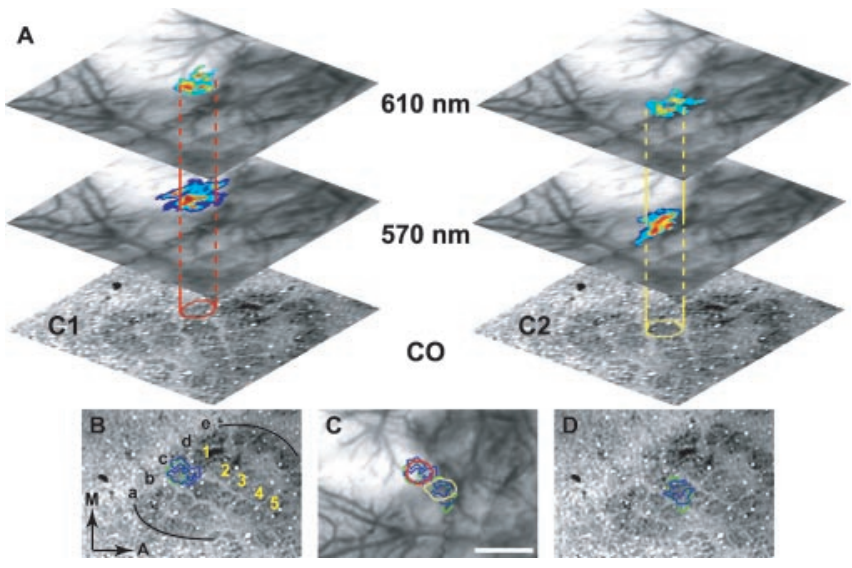

Figure 5. CBV spatial specificity and resolution. $A$, Vertical projection of the $\mathrm{C} 1$ (red) and $\mathrm{C2}$ (yellow) barrel positions identified by $\mathrm{CO}$ staining. Barrel rows (a-e) and columns (1-5) are denoted in $B$. These projections pass through statistically thresholded peak $570 \mathrm{~nm}$ (CBV) and early $610 \mathrm{~nm}$ (early oxygenation) responses overlaid on raw images, demonstrating localization of both responses with underlying anatomy. Contours enclosing the peak response area for volume (green) and early oxygenation (blue) responses are overlaid on C 0 images of $C 1(B)$ and $C 2$ (D). C, Contours for both responses and whisker representations overlaid on barrel outlines demonstrate the spatially distinct representations of both hemodynamic responses. Thus, CBV responses resolve submillimeter functional architecture with accuracy comparable with early oxygenation responses. $M$, Medial; $A$, anterior. Scale bar, $1 \mathrm{~mm}$.
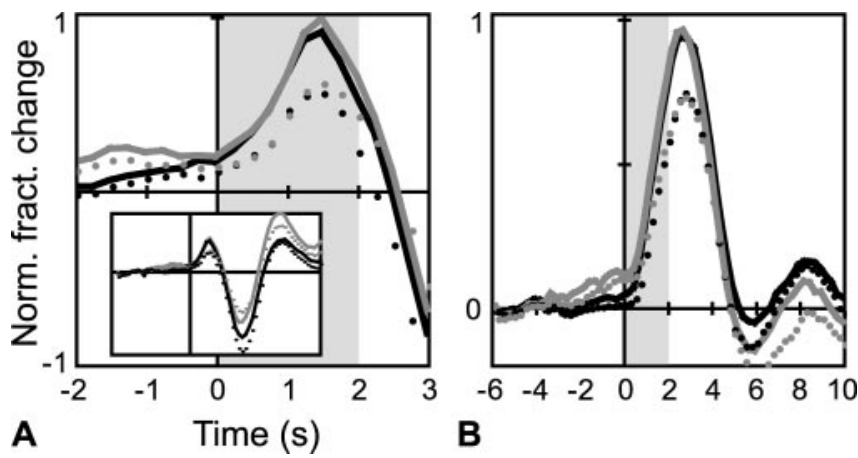

Figure 6. Vascular point spread. $A$, The $610 \mathrm{~nm}$ responses; the inset shows the full time course. $B$, The $570 \mathrm{~nm}$ responses. Black traces depict responses to C 1 stimulation measured in C1 (solid) or C2 (dotted) peak contours; gray traces are analogous for C2. Time courses were normalized to peak values in the principal barrel. Both responses extended beyond the principal column but were smaller in the neighboring column. The difference in response ratio (see Results) between the two hemodynamic signals was not significant $(p>0.2)$. The temporal profile of changes was similar in the principal and neighboring barrel.

tion between the two was much less than a barrel column diameter

In contrast, previous studies reported that CBV changes exhibited excessive vascular overspill and were, therefore, inferior to early oxygenation changes for high-resolution mapping (Frostig et al., 1990; Malonek and Grinvald, 1996). We found both volume and early oxygenation responses extended beyond neuroanatomical boundaries of principal columns, probably because of horizontal intracortical connections (Petersen et al., 2003) or passive vascular processes (Narayan et al., 1995). The

$\leftarrow$

maps of the $610 \mathrm{~nm}$ early phase $(G, I)$ are similarly reduced in statistical maps $(H, J)$. Because the $610 \mathrm{~nm}$ late phase is predominantly venous in origin, both absolute $(K, M)$ and statistical $(L, N)$ maps are biased toward large veins. Color bars apply to images above and below them. Absolute threshold scales represent fractional change $\left(\times 10^{-3}\right)$, and statistical threshold scales represent the negative $\log _{10}$ of the $p$ value. $M$, Medial; $A$, anterior. Scale bar, $1 \mathrm{~mm}$. 


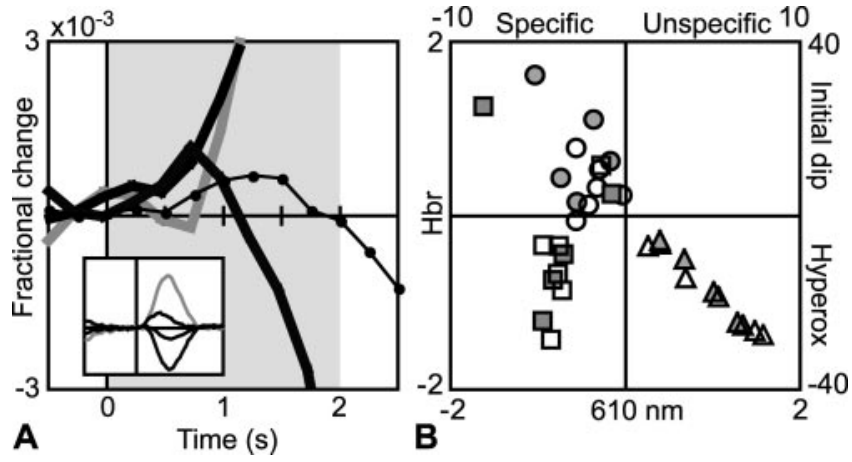

Figure 7. Etiology of spatially specific hemodynamic changes. A, Comparison between the $610 \mathrm{~nm}$ imaging time course (black circle, sign reversed for comparison) and concurrently measured $\mathrm{Hbr}$ (dark gray line), $\mathrm{HbO}_{2}$ (light gray line), and $\mathrm{Hbt}$ (black line) for one stimulation condition ( $5 \mathrm{~Hz}, 0.8 \mathrm{~mA}$; the inset shows the full time course). The $610 \mathrm{~nm}$ early phase follows $\mathrm{Hbr}$ for $0.75 \mathrm{sec}$ but then continues to increase as $\mathrm{Hbr}$ rapidly decreases. The spatially specific $610 \mathrm{~nm}$ early negative response thus incorporates both the initial dip and early hyperoxygenation. $B, H$ br versus the $610 \mathrm{~nm}$ magnitude for all stimulation conditions at $0.75 \mathrm{sec}$ (white circle, amplitude variable stimulation; gray circle, frequency variable), $1.25 \mathrm{sec}$ (white square, gray square), and $4.0 \mathrm{sec}$ (white triangle, gray triangle). The left column of the grid ( $610 \mathrm{~nm}$ early phase) thus represents spatially specific responses, and the right column (610 nm late phase) represents spatially unspecific, vascular signals. The top row $(\mathrm{Hbr},>0)$ represents the initial dip, and the bottom row $(\mathrm{Hbr},<0)$ represents hyperoxygenation. Spatial specificity can, therefore, be achieved during the initial dip or early hyperoxygenation, whereas later hyperoxygenation changes are unspecific. The axis scale values are fractional change $\times 10^{-3}$. The left and bottom scale values represent data at 0.75 and $1.25 \mathrm{sec}$, and the right and top scale values represent $4.0 \mathrm{sec}$.

temporal characteristics of hemodynamic changes over principal and adjacent columns indicate the peripheral response develops concurrently with, although to a lesser degree than, the peak response and are additional evidence for vascular spread. The response ratio between principal and neighboring barrels suggests a slightly smaller point spread function for the early oxygenation change, but the difference was not significant. Duong et al. (2001) similarly found peak CBF signals were spatially precise, although the response spread into adjacent areas. The hemodynamic point spread will depend on vascular density, which may differ across species and cortex, but our results show this spread does not necessarily preclude CBV spatial specificity.

A recent study in mouse barrel cortex found $540 \mathrm{~nm}$ optical imaging (containing mixed contributions from oxygenation and volume etiologies) emphasized vascular structures and, therefore, was not as well confined to the active barrel as the $610 \mathrm{~nm}$ early response (Erinjeri and Woolsey, 2002). This vascularity is also evident in our data for both $570 \mathrm{~nm}$ (predominantly arteriolar) and $610 \mathrm{~nm}$ (predominantly venous) images analyzed using conventional subtraction-based approaches. This point underscores the importance of statistical mapping techniques, such as the ANOVA-based approach used here, that assign higher significance to pixels in the capillary bed that respond consistently across trials and lower significance to pixels in the vasculature, the variance across trials of which is high. A similar approach was used successfully by Hess et al. (2000).

CBV response specificity supports the functional relationship suggested between microvascular architecture and neural subunits (Cox et al., 1993; Woolsey et al., 1996). According to this hypothesis, individual cortical columns are supplied by one or a few penetrating arterioles, such that flow changes localize precisely to metabolic activity. Harrison et al. (2002) observed strictures on precapillary arteriole corrosion casts, suggesting the presence of pericytes capable of controlling vascular diameter.
The opportunity for fine flow control intimated by these structures is consistent with this theory, as is the columnar specificity demonstrated with CBF fMRI (Duong et al., 2001). Our results extend these findings to volume responses, which are also predominantly arteriolar (Lee et al., 2001). Therefore, spatially precise flow regulation also leads to precise volume changes.

\section{Timing versus etiology}

We found submillimeter specificity does not require focusing on the Hbr dip; it can also be achieved during the early part of the hyperoxygenation. Combined with the CBV results, these findings suggest hemodynamic spatial specificity arises not only from a spatially localized increase in neuronal oxidative metabolism but also from highly localized changes in hemoglobin oxygenation and blood volume that accompany metabolic changes. In other words, early changes in both oxygenation (regardless of direction) and volume are spatially coupled with neuronal activity. Therefore, in the context of high-resolution functional mapping, timing (imaging early signals) is more important than etiology (CBV or oxygenation signals).

Our results indicate optimal spatial precision occurs during the first few (two to three) seconds after stimulation onset, which under our conditions included the initial dip, CBV peak, and early hyperoxygenation. Studies using oxygenation-based signals report similar spatiotemporal characteristics. Duong et al. (2000) found single-condition maps of orientation columns in primary visual cortex could only be produced using the first two seconds of the blood oxygenation level-dependent (BOLD) fMRI initial dip (which was $4 \mathrm{sec}$ in total duration). The latter $2 \mathrm{sec}$ of the dip or later hyperoxygenation failed to identify complementary orientation structure. Using subsaturating stimuli, Menon and Goodyear (1999) generated ocular dominance maps (albeit with differential imaging) from the early part of the BOLD hyperoxygenation phase. Under their conditions, they did not detect the dip, but restricting the map to early time periods still revealed submillimeter functional architecture. These findings support the hypothesis that spatial specificity depends less on response direction than timing.

The above discussion and reports from the literature suggest the following sequence of events. Neuronal activation leads to a focal increase in oxygen consumption and relative tissue $\mathrm{Hbr}$ content within a few hundred milliseconds. Synaptic activity produces increased flow, confined to the active region by fine microvascular control structures. The rapidity and magnitude of the flow response, which are a function of several physiological variables, determine the degree to which Hbr increases before being washed out. A sluggish flow response would allow local Hbr to increase detectably, whereas a fast influx would quickly diminish Hbr. In either case, the oxygenation change may still be spatially specific for the first $\sim 2-3 \mathrm{sec}$. As a byproduct of the flow response, regional CBV also increases in the feeding arterioles, with a spatial distribution similar to that of CBF. Subsequent hyperoxygenation changes $(>3 \mathrm{sec})$ propagate into draining veins away from the activation site. The exact timing of these hemodynamic processes will certainly depend on species, cortex, and physiological state, but the effects may be qualitatively similar.

This hypothesis generates testable predictions. By modulating the CBF response with pharmacological agents or alterations of physiological and anesthetic parameters, the relative size, timing, and spatial specificity of the Hbr increase or decrease can be assessed. Additionally, microvasculature CBF and CBV changes can be measured simultaneously to determine their activationinduced spatial and temporal coupling. 


\section{Implications for functional brain mapping}

Perfusion-based functional imaging techniques offering submillimeter spatial resolution are extremely useful for studying columnar cortical organization. Several studies using conventional gradient echo $\mathrm{T}_{2}{ }^{*}$ BOLD fMRI have demonstrated columnar structure, but the large venous contribution to this signal usually requires differential imaging, which presupposes common activation patterns and, therefore, limits its use (Menon et al., 1997; Menon and Goodyear, 1999; Cheng et al., 2001; Goodyear and Menon, 2001). Single-condition $\mathrm{T}_{2}$-weighted spin echo BOLD fMRI is less influenced by large veins and can resolve cortical columns, but the technique has low sensitivity and requires high ( $>4$ T) field strengths (Duong et al., 2002). Although BOLD fMRI using the initial dip has revealed submillimeter structure (Kim et al., 2000), the signal is elusive (Buxton, 2001), has a low CNR, and correlates weakly with the magnitude of changes in underlying neuronal activity (Sheth et al., 2003). CBF fMRI also demonstrates excellent spatial specificity and does not require high fields but suffers from a relatively low temporal resolution (Duong et al., 2001).

In comparison, $\mathrm{CBV}$ mapping offers several advantages. With appropriate analysis, single-condition CBV maps can resolve columnar functional organization. The increased number of arterioles supplying visual cortex columns in cats and primates compared with rat barrels suggests the capacity for even finer CBV resolution in the former. In this study, we found a fourfold and 1.5-fold increase in CNR of CBV responses relative to early and late oxygenation changes, respectively. Temporally restricting imaging to the early epoch $(2-3 \mathrm{sec})$ further increases the CNR advantage of CBV over hyperoxygenation responses because CBV peaks earlier. CBV-weighted fMRI studies have similarly reported increased CNR and parenchymal specificity of CBV signals relative to BOLD signals (Mandeville and Marota, 1999). Furthermore, functional CBV changes correlate very closely with the magnitude of evoked neuronal activity (Sheth et al., 2003). This linear correspondence is desirable in any investigation using subtly graded stimuli and is essential for cognitive subtractionbased studies. Although CBV fMRI is currently limited to animal studies because of the requirement for exogenous contrast, human trials are underway (Sharma et al., 1999).

In conclusion, we found functional CBV changes were capable of resolving columnar organization. We hypothesize that spatial localization with submillimeter accuracy is a function of temporal characteristics (using early signals) more than specific response etiology (oxygenation or volume responses). Spatial specificity requires reducing the contribution of vascular signals, which can be achieved by observing early responses and statistical thresholding. The CBV response additionally offers high CNR and linear coupling with neuronal activity, making it an attractive candidate for high-resolution mapping.

\section{References}

Britten KH (1998) Clustering of response selectivity in the medial superior temporal area of extrastriate cortex in the macaque monkey. Vis Neurosci 15:553-558.

Buxton RB (2001) The elusive initial dip. NeuroImage 13:953-958.

Buxton RB, Frank LR (1997) A model for the coupling between cerebral blood flow and oxygen metabolism during neural stimulation. J Cereb Blood Flow Metab 17:64-72.

Chen-Bee CH, Polley DB, Brett-Green B, Prakash N, Kwon MC, Frostig RD (2000) Visualizing and quantifying evoked cortical activity assessed with intrinsic signal imaging. J Neurosci Methods 97:157-173.

Cheng K, Waggoner RA, Tanaka K (2001) Human ocular dominance columns as revealed by high-field functional magnetic resonance imaging. Neuron 32:359-374.
Cooper CE, Delpy DT, Nemoto EM (1998) The relationship of oxygen delivery to absolute haemoglobin oxygenation and mitochondrial cytochrome oxidase redox state in the adult brain: a near-infrared spectroscopy study. Biochem J 332:627-632.

Cox SB, Woolsey TA, Rovainen CM (1993) Localized dynamic changes in cortical blood flow with whisker stimulation corresponds to matched vascular and neuronal architecture of rat barrels. J Cereb Blood Flow Metab 13:899-913.

Duong TQ, Kim DS, Ugurbil K, Kim SG (2000) Spatiotemporal dynamics of the BOLD fMRI signals: toward mapping submillimeter cortical columns using the early negative response. Magn Reson Med 44:231-242.

Duong TQ, Kim DS, Ugurbil K, Kim SG (2001) Localized cerebral blood flow response at submillimeter columnar resolution. Proc Natl Acad Sci USA 98:10904-10909.

Duong TQ, Yacoub E, Adriany G, Hu X, Ugurbil K, Vaughan JT, Merkle H, Kim SG (2002) High-resolution, spin-echo BOLD, and CBF fMRI at 4 and 7 T. Magn Reson Med 48:589-593.

Erinjeri JP, Woolsey TA (2002) Spatial integration of vascular changes with neural activity in mouse cortex. J Cereb Blood Flow Metab 22:353-360.

Fox PT, Raichle ME (1986) Focal physiological uncoupling of cerebral blood flow and oxidative metabolism during somatosensory stimulation in human subjects. Proc Natl Acad Sci USA 83:1140-1144.

Frostig RD, Lieke EE, Ts'o DY, Grinvald A (1990) Cortical functional architecture and local coupling between neuronal activity and the microcirculation revealed by in vivo high-resolution optical imaging of intrinsic signals. Proc Natl Acad Sci USA 87:6082-6086.

Goodyear BG, Menon RS (2001) Brief visual stimulation allows mapping of ocular dominance in visual cortex using fMRI. Hum Brain Mapp 14:210-217.

Harrison RV, Harel N, Panesar J, Mount RJ (2002) Blood capillary distribution correlates with hemodynamic-based functional imaging in cerebral cortex. Cereb Cortex 12:225-233.

Hess A, Stiller D, Kaulisch T, Heil P, Scheich H (2000) New insights into the hemodynamic blood oxygenation level-dependent response through combination of functional magnetic resonance imaging and optical recording in gerbil barrel cortex. J Neurosci 20:3328-3338.

Hubel DH, Wiesel TN (1959) Integrative action in the cat's lateral geniculate body. J Physiol (Lond) 148:574-591.

Jones M, Berwick J, Johnston D, Mayhew J (2001) Concurrent optical imaging spectroscopy and laser-Doppler flowmetry: the relationship between blood flow, oxygenation, and volume in rodent barrel cortex. NeuroImage 13:1002-1015.

Kim DS, Duong TQ, Kim SG (2000) High-resolution mapping of isoorientation columns by fMRI. Nat Neurosci 3:164-169.

Lee SP, Duong TQ, Yang G, Iadecola C, Kim SG (2001) Relative changes of cerebral arterial and venous blood volumes during increased cerebral blood flow: implications for BOLD fMRI. Magn Reson Med 45:791-800.

Lindauer U, Royl G, Leithner C, Kuhl M, Gold L, Gethmann J, Kohl-Bareis M, Villringer A, Dirnagl U (2001) No evidence for early decrease in blood oxygenation in rat whisker cortex in response to functional activation. NeuroImage 13:988-1001.

Malonek D, Grinvald A (1996) Interactions between electrical activity and cortical microcirculation revealed by imaging spectroscopy: implications for functional brain mapping. Science 272:551-554.

Malonek D, Dirnagl U, Lindauer U, Yamada K, Kanno I, Grinvald A (1997) Vascular imprints of neuronal activity: relationships between the dynamics of cortical blood flow, oxygenation, and volume changes following sensory stimulation. Proc Natl Acad Sci USA 94:14826-14831.

Mandeville JB, Marota JJ (1999) Vascular filters of functional MRI: spatial localization using BOLD and CBV contrast. Magn Reson Med 42:591-598.

Masino SA, Kwon MC, Dory Y, Frostig RD (1993) Characterization of functional organization within rat barrel cortex using intrinsic signal optical imaging through a thinned skull. Proc Natl Acad Sci USA 90:9998-10002.

Mayhew JE, Askew S, Zheng Y, Porrill J, Westby GW, Redgrave P, Rector DM, Harper RM (1996) Cerebral vasomotion: a $0.1-\mathrm{Hz}$ oscillation in reflected light imaging of neural activity. NeuroImage 4:183-193.

Menon RS, Goodyear BG (1999) Submillimeter functional localization in human striate cortex using BOLD contrast at 4 Tesla: implications for the vascular point-spread function. Magn Reson Med 41:230-235.

Menon RS, Ogawa S, Strupp JP, Ugurbil K (1997) Ocular dominance in human V1 demonstrated by functional magnetic resonance imaging. J Neurophysiol 77:2780-2787. 
Mountcastle VB (1957) Modality and topographic properties of single neurons in cat's somatic sensory cortex. J Neurophysiol 20:408-434.

Nakai T, Nishimura G, Yamamoto K, Tamura M (1997) Expression of optical diffusion coefficient in high-absorption turbid media. Phys Med Biol 42:2541-2549.

Narayan SM, Esfahani P, Blood AJ, Sikkens L, Toga AW (1995) Functional increases in cerebral blood volume over somatosensory cortex. J Cereb Blood Flow Metab 15:754-765.

Obrig H, Villringer A (2003) Beyond the visible-imaging the human brain with light. J Cereb Blood Flow Metab 23:1-18.

Petersen CC, Grinvald A, Sakmann B (2003) Spatiotemporal dynamics of sensory responses in layer $2 / 3$ of rat barrel cortex measured in vivo by voltage-sensitive dye imaging combined with whole-cell voltage recordings and neuron reconstructions. J Neurosci 23:1298-1309.

Rakic P (2002) Evolving concepts of cortical radial and areal specification. Prog Brain Res 136:265-280.

Sato C, Nemoto M, Tamura M (2002) Reassessment of activity-related optical signals in somatosensory cortex by an algorithm with wavelengthdependent path length. Jpn J Physiol 52:301-312.

Sharma R, Saini S, Ros PR, Hahn PF, Small WC, de Lange EE, Stillman AE, Edelman RR, Runge VM, Outwater EK, Morris M, Lucas M (1999) Safety profile of ultrasmall superparamagnetic iron oxide ferumoxtran10: phase II clinical trial data. J Magn Reson Imaging 9:291-294.

Sheth S, Nemoto M, Guiou M, Walker M, Pouratian N, Toga AW (2003) Evaluation of coupling between optical intrinsic signals and neuronal activity in rat somatosensory cortex. NeuroImage 19:884-894.

Shoham D, Grinvald A (2001) The cortical representation of the hand in macaque and human area S-I: high resolution optical imaging. J Neurosci 21:6820-6835
Slovin H, Arieli A, Hildesheim R, Grinvald A (2002) Long-term voltagesensitive dye imaging reveals cortical dynamics in behaving monkeys. J Neurophysiol 88:3421-3438.

Takashima I, Kajiwara R, Iijima T (2001) Voltage-sensitive dye versus intrinsic signal optical imaging: comparison of optically determined functional maps from rat barrel cortex. NeuroReport 12:2889-2894.

Thompson JK, Peterson MR, Freeman RD (2003) Single-neuron activity and tissue oxygenation in the cerebral cortex. Science 299:1070-1072.

Ts'o DY, Frostig RD, Lieke EE, Grinvald A (1990) Functional organization of primate visual cortex revealed by high resolution optical imaging. Science 249:417-420.

Ugurbil K, Toth L, Kim DS (2003) How accurate is magnetic resonance imaging of brain function? Trends Neurosci 26:108-114.

Villringer A, Dirnagl U (1995) Coupling of brain activity and cerebral blood flow: basis of functional neuroimaging. Cereb Brain Metab Rev 7:240-276.

Welker C (1971) Microelectrode delineation of fine grain somatotopic organization of $(\mathrm{SmI})$ cerebral neocortex in albino rat. Brain Res 26:259-275.

Wong-Riley MT, Welt C (1980) Histochemical changes in cytochrome oxidase of cortical barrels after vibrissal removal in neonatal and adult mice. Proc Natl Acad Sci USA 77:2333-2337.

Woolsey TA, Van der Loos H (1970) The structural organization of layer IV in the somatosensory region (SI) of mouse cerebral cortex. Brain Res 17:205-242.

Woolsey TA, Rovainen CM, Cox SB, Henegar MH, Liang GE, Liu D, Moskalenko YE, Sui J, Wei L (1996) Neuronal units linked to microvascular modules in cerebral cortex: response elements for imaging the brain. Cereb Cortex 6:647-660. 